\title{
The Association between Dietary Diversity Score and Somatoform Complaints Profile among Iranian Adults
}

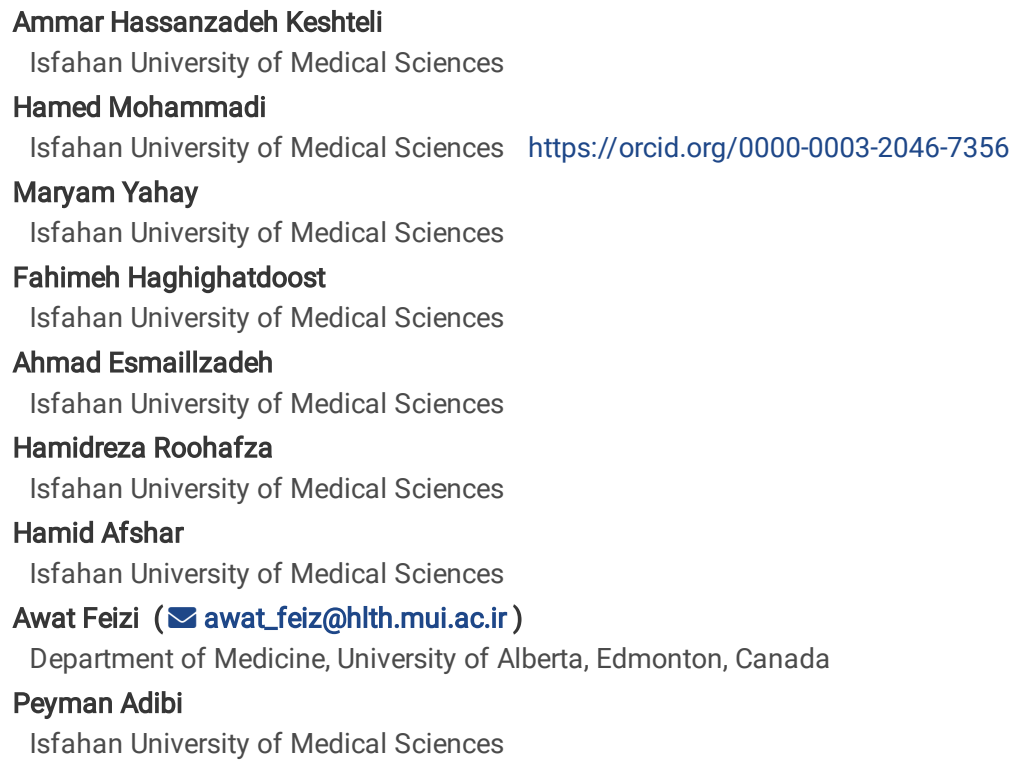




\section{Abstract}

Background: The present study aimed to investigate the association between dietary diversity score (DDS) and somatoform complaints profile.

Methods: The current cross-sectional study was conducted on 2818 adults aged 19-65 years working in Isfahan province, Iran. A Dish-based 106-item Semiquantitative Food Frequency Questionnaire (DS-FFQ) was used to collect dietary intake of participants. Somatoform symptoms were assessed using a validated comprehensive 31-items questionnaire.

Results: After adjusting for the impacts of various confounders it was revealed that lower DDS is associated with higher risk of experiencing greater scores of psychological $(\mathrm{OR}=1.44,95 \% \mathrm{Cl}: 1.06,1.95 ; \mathrm{P}=0.029)$ and pharyngeal-respiratory ( $\mathrm{OR}=1.54,95 \% \mathrm{Cl}: 1.17,2.04 ; \mathrm{P}=0.009)$ somatoform complaints. No significant association was found between DDS and neuro-skeletal and gastrointestinal somatoform complaints. In addition, stratified analysis by sex indicated that the lower DDS increased the risk of higher scores of psychological $(\mathrm{OR}=1.73,95 \% \mathrm{Cl}=1.17,2.56 ; \mathrm{P}=0.02)$ and pharyngeal-respiratory $(\mathrm{OR}=$ $1.75,95 \% \mathrm{Cl}=1.22,2.49, \mathrm{P}=0.007)$ somatic complaints in women, but not in men.

Conclusion: Present study indicated that the lower DDS is associated with higher risk of psychological and pharyngeal-respiratory somatoform complaints. Future prospective studies and particularly clinical trials are warranted to confirm these findings.

\section{Background}

Somatoform complaints are refer to spectrum of non-specific physical symptoms that cannot be fully clarified by a known medical condition (1). Previous reports indicated that 25-50 percent of primary care patients suffer from somatoform complaints (2). Lower socioeconomic status, family history of chronic illnesses, female sex, concurrent general medical disorders and history of childhood chronic illnesses are the main risk factors for somatoform complaints (3, 4). Also, several studies have settled a close relationship between psychiatric disorders and somatoform complaints $(5,6)$. It has been reported that severity of somatoform complaints has positive association with incidence of mental health problems (7). In addition, some studies have established that patients with depression or anxiety exhibit more than twice somatoform complaints compared to general population $(8,9)$.

Several studies have shown that poor diet quality is a modifiable risk factor for mental problems (10-12). Observational studies have indicated a negative association between single nutrients or foods like B vitamins, omega 3 fatty acids, fruits, vegetables, fish, and whole grains and risk of depression (13, 14). Compared to the intake of single nutrients or foods, considering the general pattern of diet to assess diet quality could provide a better insight into dietdisease relations and may be more predictive of chronic disease risk (15). In this context, several studies have investigated the association between diet quality and psychiatric disorders $(16,17)$. Dietary diversity score (DDS), as an indicator of diversity within food groups, is a reliable diet quality index (18). Available studies have indicated an inverse relationship between DDS and psychological disorders such as depression (19), anxiety (20) and a decline in intellectual activity (21). Moreover, findings from a recent meta-analysis of observational studies have revealed a significant inverse association between healthy dietary pattern and risk of depression (22).

With regard to close relationship between psychiatric disorders and somatic symptoms, this may be an effective strategy to evaluate the association between diet quality and somatoform complaints. To our knowledge, there is no study examining the association between quality of diet and somatoform complaints. Moreover, low consumption of fruits and vegetables and high intakes of refined grains along with lack of alcohol intake in Middle-Eastern population makes their dietary pattern of great interest for the assessment of diet-disease relations. Hence, we aimed to investigate the association of DDS, as a diet quality indicator, with somatoform complaints in a large cross-sectional population based study.

\section{Subjects And Methods}

\section{Participants}

The current cross-sectional study was carried out within the framework of the Study on the Epidemiology of Psychological- Alimentary Health and Nutrition (SEPAHAN). The SEPAHAN project was conducted in two phases in a large population of Iranian adults in Isfahan province in the central part of Iran. Details about SEPAHAN have been reported previously (23). SEPAHAN was conducted in two phases. In the first phase, questionnaires were distributed among 10,087 people aged 19-65 years, and 8691 subjects returned the completed questionnaires (response rate $86.16 \%$ ). At the second phase, questionnaires with focus on common gastrointestinal, psychological, and personality traits were distributed and 6239 questionnaires were completed (response rate: $64.64 \%$ ). After merging data from these two phases, complete information was available for 4763 people. We excluded participants with missing data (outcome and covariate variables), women with pregnancy and lactation, and those with under- or over-estimation of energy intake ( $<800$ or $>4200 \mathrm{kcal} / \mathrm{day})$. Finally, data on 2818 subjects used in the current analysis. Written informed consent forms were completed by all participants before enrolment. The Bioethics Committee of Isfahan University of Medical Sciences, Isfahan, Iran, approved this study (\#189069, \#189082, and \#189086) (23).

\section{Dietary assessment}

Dietary data were collected using a Willett-format (24) Dish-based 106-item Semi-quantitative Food Frequency Questionnaire (DS-FFQ), which was designed and validated specifically for Iranian adults (25). Briefly, the DS-FFQ contained five categories of foods and dishes: (1) mixed dishes (2) carbohydrate-based foods (3) dairy products (4) fruits and vegetables; and (5) miscellaneous food items and beverages (including sweets, fast foods, nuts, desserts and beverages). Common portion sizes and the average reported frequency were used to determine the amount of food consumed. Daily intakes of all food items were computed and converted to $\mathrm{g} / \mathrm{d}$ using household measures (26). In addition, we calculated daily intakes of 38 nutrients (and bioactive substances) for each participant using the US Department of Agriculture's (USDA) national nutrient databank (27). 


\section{Dietary Diversity Score Estimation}

DDS was calculated using Kant et al. method (28). Briefly, DDS consisted of five food groups which divided into twenty-three subgroups: bread-grain (refined bread, biscuits, macaroni, whole grain bread, corn flakes, rice and refined flour), fruit (fruit and fruit juice, berries and citrus), vegetables (vegetables, potato, tomato, other starchy vegetables, legumes, yellow vegetables and green vegetables), meat (red meat, poultry, fish and eggs) and dairy (milk, yoghurt and cheese). Based on scoring criteria, each food group receives a diversity score between 0-2, and total DDS score would range from 0 to 10 . To be counted as a 'consumer' for any of the food group categories, a respondent had to consume at least one-half of the serving of any subgroup in a day as defined by the Food Pyramid quantity criteria. For example, in the vegetables group, if a person consumed legumes and green vegetables, his or her score was calculated as $(2 \div 7)$ $x 2=0.57$. Therefore, the diversity score of vegetables group would be 0.57 .

\section{Assessment of Somatoform Symptoms}

There was not a separate questionnaire to examine somatoform symptoms in the SEPAHAN study. However, we settled a validated questionnaire based on 31 items in SEPAHAN's questionnaires common with the 47-items questionnaire used in the Lacourt et al.'s study (29), and "the patient health questionnaire (PHQ)" (30) as valid and standard tools for the assessment of somatoform symptoms. The reliability of this instrument has been evaluated previously (31) and showed a strong internal reliability with a Cronbach's alpha score of 0.903 . The 31-items questionnaire was used to examine the frequency of somatoform symptoms. Based on a four points Likert scale (never, sometimes, often, and always) each subject determined the frequency of experiencing each symptom in the past three months. For one item (Dry mouth), the rating scale was as: never, low and high.

\section{Assessment of other variables}

Self-administered questionnaires were distributed to collect information on age, sex, weight, height, marital status (married, single), education levels ( $\leq 12$ yrs, 12-16 yrs, >16 yrs), smoking habits (non-smoker/former smoker/current smoker), disease history (hyperlipidemia, hypertension, diabetes mellitus, asthma, stroke, myocardial infarction, gastrointestinal bleeding, gallstone, cancer, Crohn's disease and ulcerative colitis), current use of anti-psychothropic medications and dietary supplements. Body mass index (BMI) was computed as weight $(\mathrm{kg})$ divided by height ${ }^{2}\left(\mathrm{~m}^{2}\right)$. Overweight and obesity were defined as BMI=25-30 and BMI>30, respectively. General Practice Physical Activity Questionnaire (GPPAQ) was used to assess the physical activity status of participants (32). According to GPPAQ, participants were divided into inactive or moderately inactive and moderately active or active categories. Stressful life events questionnaire was used to assess the stressful life events over the past 6 months (33). Total stress score ranged from 0 to 83 , with higher scores indicating more sever stressful life events.

\section{Statistical analysis}

Exploratory factor analysis using principal component extraction approach was used to identify profiles of somatoform complaints based on the 31 individual somatoform symptoms. The orthogonal varimax rotation procedure was used to find the interpretable factors. Factors were retained for further analysis based on their natural interpretation and eigenvalues on the Scree plot. In this study, we retained factors with eigenvalues $>2$ as this cut-off could resulted in more interpretable somatoform complaints profiles and explain sufficient amounts of total variance. Four main somatoform complaints profiles were labelled based on the loaded somatoform symptoms in each factor. The factor score for each profile was computed by summing up items of somatoform complaints weighted by their factor loadings and assigned into each participant (Table 1) (31), and then based on the median value were categorized into high or low score of each factor. We also classified the participants based on tertiles of DDS. Continuous and categorical demographic variables were compared across tertiles of DDS using analysis of variance and Chi-square tests, respectively. Analysis of covariance was used for assessment of age-, sex- and energyadjusted intakes of foods and nutrients across quartiles of DDS. The association of DDS with being in the higher than median of somatoform complaints profiles scores was assessed using univariate and multivariable logistic regression in the crude and different adjusted models. First, adjustments were done for age, sex and energy intake. In the second model, we further controlled for marital status (single/married), education ( $\leq 12 \mathrm{yrs}, 12-16 \mathrm{yrs},>16 \mathrm{yrs}$ ), smoking status (non-smoker/former smoker/current smoker), physical activity (inactive/ moderately inactive vs. moderately active/active) and BMI (continuous). Ultimately, the effects of stressful life event (continuous), anti-psychotic medicine (yes/no) and medical condition were additionally adjusted in model 3. All these analyses were done using analysis of covariance with Bonferroni correction. To calculate the trend of OR across increasing tertiles of DDS, we considered DDS tertiles as an ordinal variable. In all analyses, the third tertile of the DDS was considered as the reference category and $p$ for linear trends was determined using Mantel-Haenszel extension of chi-square test. Stratified analyses by sex, applying the above-mentioned adjusted models, was run to examine potential modifying effect of sex in relation to the association of DDS and somatoform complaints profiles. All statistical analyses were done using the Statistical Package for Social Sciences (SPSS, version 16.0 for Windows, 2006, SPSS, Inc, Chicago, IL). P value <0.05 was considered statistically significant.

\section{Results}

Based on results of factor analysis using principal component extraction approach on the 31 individual somatoform complaints, four factors were extracted and labeled as psychological, gastrointestinal, neuro-skeletal, and pharyngeal- respiratory (Table 1 ). They accounted for $12.70 \%, 11.73 \%, 11.55 \%$, and $8.85 \%$ of the total variance, respectively (31).

General characteristics and somatoform complaints profiles of study participants across tertiles of DDS are presented in Table 2. Participants in the lowest tertile of DDS had lower educational levels $(p<0.0001)$ and were more likely to be overweight or obese $(p<0.0001)$. Compared to the upper tertile, those who were assigned into the lowest tertile of DDS had higher scores of psychological $(p=0.004)$, gastrointestinal $(p=0.02)$, and pharyngeal-respiratory $(0.001)$ symptoms. Means of age and BMI were not different across the tertiles of DDS and sex, marital status, physical activity, smoking status and Neuro-skeletal symptoms were similarly distributed across the tertiles of DDS $(p>0.05)$.

Page $3 / 11$ 
Table 3 shows age-, sex- and energy-adjusted of mean dietary intakes and food groups of study participants across median cuts of somatoform complaints profiles. Compared with those who had scores lower than median of psychological symptoms, those with higher than median had higher intake of caffeine and lower consumption of fiber, magnesium, vitamin B6, overall DDS and DDS within vegetables, fruit and meat groups. Furthermore, subjects who had scores greater than median of gastrointestinal symptoms had higher consumption of caffeine and lower intakes of energy, protein, fiber, folate, magnesium, vitamin B6, vitamin B12, overall DDS and DDS within vegetables, fruit and meat groups compared with those who were in the lower than median. Subjects in the higher than median of neuro-skeletal symptoms scores had lower intakes of fiber, magnesium, overall DDS and DDS within fruit group compared with those who were in the lower than median scores category. In addition, individuals with scores higher than median of pharyngeal-respiratory symptoms had lower overall DDS and DDS within vegetables, fruit and dairy groups.

Crude and multivariable-adjusted odds ratios (OR) for the associations of DDS with somatoform complaints profiles in the entire study population are presented in Table 4. Individuals in the first tertile of DDS had greater risk for being in higher scores of psychological $(\mathrm{OR}=1.37,95 \% \mathrm{Cl}$ : $1.14,1.66$; $\mathrm{P}=0.004)$, gastrointestinal $(O R=1.33,95 \% \mathrm{Cl}: 1.08,1.66 ; \mathrm{P}=0.02)$ and pharyngeal-respiratory $(\mathrm{OR}=1.39,95 \% \mathrm{Cl}: 1.15,1.68 ; \mathrm{P}=0.001)$ somatoform complaints level in comparison with those in the top tertile in the crude model. Adjustment for age, sex, energy intake, lifestyle factors, BMI, stressful life events, medical condition and anti-psychotropic medicines strengthened the risk of having higher scores of psychological $(\mathrm{OR}=1.44,95 \% \mathrm{Cl}$ : $1.06,1.95 ; \mathrm{P}=0.029)$ and pharyngealrespiratory $(\mathrm{OR}=1.54,95 \% \mathrm{Cl}: 1.17,2.04 ; \mathrm{P}=0.009)$ somatoform complaints, for those in first tertile of DDS compared with subjects in top tertile. However, controlling for various confounders eliminated the significant association between DDS and gastrointestinal somatoform complaints $(\mathrm{OR}=1.17,95 \% \mathrm{Cl}=$ $0.85-1.60 ; P=0.4)$. There were no significant association between DDS and neuro-skeletal somatoform complaints either in the crude or in the adjusted models.

Stratified analysis by sex (Table 5) indicated an inverse associations between DDS and psychological somatoform complaints (OR = $1.73,95 \% \mathrm{Cl}=1.17,2.56$; $\mathrm{P}=0.02$ ) in women, but not in men. Greater risk of having pharyngeal-respiratory somatoform complaints was observed among men and women, in the crude model. However, after controlling for potential confounders, these associations were eliminated in men, whilst remained significant in all models for women. No significant association was found between DDS and gastrointestinal and neuro-skeletal somatoform complaints in the crude and all adjusted models in women. Men in the first tertile of DDS had greater risk for having higher scores of gastrointestinal and neuro-skeletal somatoform complaints in the crude model, but the significance was disappeared in the fully adjusted model.

\section{Discussion}

To the best of our knowledge, this is the first study examining the association between DDS and somatoform complaints. The results of present crosssectional study showed that lower DDS was related to higher odds of psychological and pharyngeal-respiratory somatoform complaints. In addition, stratified analysis by sex revealed a significant association between lower tertile of DDS and higher risk of psychological and pharyngeal-respiratory somatoform complaints in women, but not in men.

Somatoform complaints are physical symptoms which manifest the psychological illnesses (34). Patients with somatoform complaints are one of the most attenders of primary care and have high overall health care utilization (35). Hence, identification of potential risk factors is essential in management of somatoform complaints. Diet is one of the well-known risk factors for psychological disorders (22). Although associations between nutrients and dietary patterns and mental disorders (e.g. depression, anxiety and stress) have been shown in previous studies $(16,22,36)$, no evidence is available linking diet quality indices and risk of somatoform complaints.

In the present study, for the first time, we showed that low diet quality, identified by low DDS, is a risk factor for psychological and pharyngeal-respiratory somatoform complaints. In line with our findings, Poorrezaeian et al. showed an inverse association between DDS and depression (19) and anxiety (20) in women. However, they did not observe any significant association between DDS and stress. In another study, Akbaraly et al. (36) showed that greater adherence to Alternate Healthy Eating Index )AHEI (was associated with lower risks of recurrent depressive symptoms. Moreover, people with high diet quality, identified by AHEI-2010, had 49\% and 45\% lower chance for anxiety and depression, respectively (16).

It has been reported that a healthy dietary pattern (characterized by high consumption of fruits, vegetables, nuts, legumes, fish and olive oil) was associated with lower risk of depression (37). Traditional dietary pattern with high content of fruits, vegetables, whole grains, fish and meat have found to be inversely associated with depression and anxiety in women (38). In addition, Western dietary pattern which is characterized by higher intake of processed foods, beverage, sweets, red meat and its products was associated with higher risks of depression and anxiety $(39,40)$. Recent meta-analysis suggested that healthy dietary pattern may decrease the risk of depression, whereas Western dietary pattern may increase the risk of depression (41).

In the present study, subjects in the higher median of psychological and pharyngeal-respiratory somatoform symptoms significantly had lower DDS within fruit, vegetables, dairy and meat groups compared to the subjects in the lower median. In line with our findings, McMartin et al. (42) suggested an inverse relationship between fruit and vegetable intake with mental disorders. In another study, Mikolajczyk et al. (43) showed that lower consumption of fruits, vegetables and meat was positively associated with depressive symptoms in female students. In addition, an inverse association was observed between higher intakes of meat, poultry and vegetables and depression in Australian adults (44). The protective role of the low fat dairy intake on cognitive health has been shown in a cross-sectional study (45). Moreover, some other studies have shown an inverse association between dairy products consumption and risk of mental disorders $(46,47)$.

The inverse association between DDS and psychological and pharyngeal-respiratory somatoform symptoms might be explained by some mechanisms. With regard to the key role of oxidative stress in mental disorders, it has been reported that higher DDS are associated with higher blood levels of antioxidant enzymes (e.g. glutathione peroxidase, superoxide dismutase) (48), which could attenuate neuronal damage of oxidative stress. Also, higher DDS is associated with higher intake of anti-inflammatory foods and nutrients (such as fruits and vegetables, legumes, nuts, vitamin A, magnesium and B vitamins) (19) which 
could reduce the risk of cardiovascular diseases and inflammation as a well-known risk factors of psychological disorders (49, 50). Moreover, it has been suggested that serum proinflammatory cytokines are involved in the development of inflammatory and neuropathic pain (51), therefore it could be expected that anti-inflammatory properties of diverse diets would ameliorate neuropathic pain by modulating proinflammatory cytokines levels. In addition, dietary diversity is positively associated with nutrient adequacy of the diet $(52,53)$, which could inversely associated with psychological symptoms $(54,55)$.

In the present study, we observed a gender-specific association between DDS and somatoform complaints. Lower prevalence of somatoform complaints in men compared with female might explain this non-significant association (3). In addition, hormonal fluctuations, specifically estrogen, in triggering depressive symptoms in women is could be another explanation (56). Moreover, accuracy of reported dietary intake and food preferences might be different between men and women $(50,57)$.

A major strength of present study is the large included population and adjustments for several potential confounders. In addition, we evaluated the dietary intakes by DS-FFQ, which provides more accurate information than a semi-quantitative FFQ (23). The present study had some limitations that should be considered in interpretation of results. With regard to the cross-sectional design of the study, we could not confer a causal link between DDS and somatoform complaints. Hence, prospective studies are required to determine a causal relationship. We used a validated FFQ to evaluate usual dietary intake, which is depending on memory and could increase the rate of misclassification. In addition, since SEPAHAN study was conducted on adults working in health centres across Isfahan province, generalization of our findings to all Iranian population should be done with caution.

\section{Conclusion}

In conclusion, we found evidence indicating that lower DDS is associated with higher odds of psychological and pharyngeal-respiratory somatoform complaints. However, after subgroup analysis by sex, these associations were eliminated in men, whilst remained significant in women. These initial findings highlight the crucial role of diet characterized by higher diversity in mental health. Future prospective studies are warranted to confirm these findings.

\section{Declarations}

\section{Authors' contributions}

$\mathrm{HM}$ and MY contributed to data interpretation and manuscript drafting; $\mathrm{AF}$ and $\mathrm{FH}$ contributed to statistical analysis, data interpretation and manuscript drafting; EA KAH, RH, HA and AP contributed to SEPAHAN study concepts and design, data collection and drafting of the manuscript; Feizi A supervised the current secondary study; all authors approved the final version of the manuscript.

\section{Acknowledgements}

We are grateful to thank all staff of Isfahan University of Medical Sciences (IUMS) who kindly participated in our study, and the staff of the Public Relations Unit, and other authorities of IUMS for their excellent cooperation.

\section{Competing interests}

The authors declare that they have no competing interests.

\section{Availability of data and materials}

The datasets used and/or analyzed during the current study are available from the corresponding author on reasonable request.

\section{Consent for publication}

Not applicable.

\section{Ethics approval and consent to participate}

The study protocol was approved by the Ethics Committee of Isfahan University of Medical Sciences.

\section{Financial support}

SEPAHAN was financially supported by a grant from the Vice Chancellery for Research and Technology, IUMS (Project No. 189069, 189082, and 189086).

\section{References}

1. Kroenke K, Rosmalen JG. Symptoms, syndromes, and the value of psychiatric diagnostics in patients who have functional somatic disorders. Medical Clinics. 2006;90(4):603-26.

2. Hilderink P, Collard R, Rosmalen J, Voshaar RO. Prevalence of somatoform disorders and medically unexplained symptoms in old age populations in comparison with younger age groups: a systematic review. Ageing research reviews. 2013;12(1):151-6.

3. Creed F, Barsky A. A systematic review of the epidemiology of somatisation disorder and hypochondriasis. Journal of psychosomatic research. 2004;56(4):391-408. 
4. Creed FH, Davies I, Jackson J, Littlewood A, Chew-Graham C, Tomenson B, et al. The epidemiology of multiple somatic symptoms. Journal of psychosomatic research. 2012;72(4):311-7.

5. Bair MJ, Robinson RL, Katon W, Kroenke K. Depression and pain comorbidity: a literature review. Archives of internal medicine. 2003;163(20):2433-45.

6. Kapfhammer H-P. Somatic symptoms in depression. Dialogues in clinical neuroscience. 2006;8(2):227.

7. Shabbeh Z, Feizi A, Afshar H, Hassanzade Kashtali A, Adibi P. Identifying the profiles of psychosomatic disorders in an iranian adult population and their relation to psychological problems. Journal of Mazandaran University of Medical Sciences. 2016;26(137):82-94.

8. Katon W, Sullivan M, Walker E. Medical symptoms without identified pathology: relationship to psychiatric disorders, childhood and adult trauma, and personality traits. Annals of internal medicine. 2001;134(9_Part_2):917-25.

9. Simon GE, VonKorff M, Piccinelli M, Fullerton C, Ormel J. An international study of the relation between somatic symptoms and depression. New England Journal of Medicine. 1999;341(18):1329-35.

10. Akbaraly TN, Brunner EJ, Ferrie JE, Marmot MG, Kivimaki M, Singh-Manoux A. Dietary pattern and depressive symptoms in middle age. The British Journal of Psychiatry. 2009;195(5):408-13.

11. Park J-Y, You J-S, Chang K-J. Dietary taurine intake, nutrients intake, dietary habits and life stress by depression in Korean female college students: a casecontrol study. Journal of biomedical science. 2010;17(1):S40.

12. Quirk SE, Williams LJ, O'Neil A, Pasco JA, Jacka FN, Housden S, et al. The association between diet quality, dietary patterns and depression in adults: a systematic review. BMC psychiatry. 2013;13(1):175.

13. Woo J, Lynn H, Lau W, Leung J, Lau E, Wong S, et al. Nutrient intake and psychological health in an elderly Chinese population. International journal of geriatric psychiatry. 2006;21(11):1036-43.

14. Khosravi M, Sotoudeh G, Raisi F, Majdzadeh R, Foroughifar T. Comparing dietary patterns of depressed patients versus healthy people in a case control protocol. BMJ open. 2014;4(2):e003843.

15. Hu FB. Dietary pattern analysis: a new direction in nutritional epidemiology. Current opinion in lipidology. 2002;13(1):3-9.

16. Saneei P, Hajishafiee M, Keshteli AH, Afshar H, Esmaillzadeh A, Adibi P. Adherence to Alternative Healthy Eating Index in relation to depression and anxiety in Iranian adults. British Journal of Nutrition. 2016;116(2):335-42.

17. Beydoun MA, Kuczmarski MTF, Mason MA, Ling SM, Evans MK, Zonderman AB. Role of depressive symptoms in explaining socioeconomic status disparities in dietary quality and central adiposity among US adults: a structural equation modeling approach-. The American journal of clinical nutrition. 2009;90(4):1084-95.

18. Kant AK, Graubard BI. A comparison of three dietary pattern indexes for predicting biomarkers of diet and disease. Journal of the American College of Nutrition. 2005;24(4):294-303.

19. Poorrezaeian M, Siassi F, Milajerdi A, Qorbani M, Karimi J, Sohrabi-Kabi R, et al. Depression is related to dietary diversity score in women: a cross-sectional study from a developing country. Ann Gen Psychiatry. 2017;16:39.

20. Poorrezaeian M, Siassi F, Qorbani M, Karimi J, Koohdani F, Asayesh H, et al. Association of dietary diversity score with anxiety in women. Psychiatry Res. 2015;230(2):622-7.

21. Otsuka R, Kato Y, Nishita Y, Tange C, Nakamoto M, Tomida M, et al. Dietary diversity and 14-year decline in higher-level functional capacity among middleaged and elderly Japanese. Nutrition. 2016;32(7):784-9.

22. Lai JS, Hiles S, Bisquera A, Hure AJ, McEvoy M, Attia J. A systematic review and meta-analysis of dietary patterns and depression in community-dwelling adults-. The American journal of clinical nutrition. 2013;99(1):181-97.

23. Adibi P, Keshteli AH, Esmaillzadeh A, Afshar H, Roohafza H, Bagherian-Sararoudi R, et al. The study on the epidemiology of psychological, alimentary health and nutrition (SEPAHAN): overview of methodology. Journal of Research in Medical Sciences. 2012;17.

24. Willett W. Nutritional epidemiology: Oxford University Press; 2012.

25. Keshteli AH, Esmaillzadeh A, Rajaie S, Askari G, Feinle-Bisset C, Adibi P. A dish-based semi-quantitative food frequency questionnaire for assessment of dietary intakes in epidemiologic studies in Iran: design and development. International journal of preventive medicine. 2014;5(1):29.

26. Ghaffarpour M, Houshiar-Rad A, Kianfar H. The manual for household measures, cooking yields factors and edible portion of foods. Tehran: Nashre Olume Keshavarzy. 1999;7:213.

27. Kimura Y, Wada T, Okumiya K, Ishimoto Y, Fukutomi E, Kasahara Y, et al. Eating alone among community-dwelling Japanese elderly: association with depression and food diversity. The journal of nutrition, health \& aging. 2012;16(8):728-31.

28. Kant AK, Schatzkin A, Ziegler RG. Dietary diversity and subsequent cause-specific mortality in the NHANES I epidemiologic follow-up study. Journal of the American College of Nutrition. 1995;14(3):233-8.

29. Lacourt T, Houtveen J, van Doornen L. "Functional somatic syndromes, one or many?": An answer by cluster analysis. Journal of psychosomatic research. 2013;74(1):6-11.

30. Spitzer RL, Kroenke K, Williams JB, Group PHQPCS. Validation and utility of a self-report version of PRIME-MD: the PHQ primary care study. Jama. 1999;282(18):1737-44.

31. Heidari Z, Keshteli AH, Feizi A, Afshar H, Adibi P. Somatic complaints are significantly associated with chronic uninvestigated dyspepsia and its symptoms: A large cross-sectional population based study. Journal of neurogastroenterology and motility. 2017;23(1):80.

32. for Nursing NCC. The General Practice Physical Activity Questionnaire (GPPAQ). 2008. 
33. Roohafza H, Ramezani M, Sadeghi M, Shahnam M, Zolfagari B, Sarafzadegan N. Development and validation of the stressful life event questionnaire. International journal of public health. 2011;56(4):441-8.

34. Wong JY-H, Fong DY-T, Chan KK-W. Anxiety and insomnia as modifiable risk factors for somatic symptoms in Chinese: a general population-based study. Quality of Life Research. 2015;24(10):2493-8.

35. Hiller W, Fichter MM. High utilizers of medical care. Journal of Psychosomatic Research. 2004;56(4):437-43.

36. Akbaraly TN, Sabia S, Shipley MJ, Batty GD, Kivimaki M. Adherence to healthy dietary guidelines and future depressive symptoms: evidence for sex differentials in the Whitehall II study-. The American journal of clinical nutrition. 2013;97(2):419-27.

37. Rashidkhani B, Gargari BP, Ranjbar F, Zareiy S, Kargarnovin Z. Dietary patterns and anthropometric indices among Iranian women with major depressive disorder. Psychiatry research. 2013;210(1):115-20.

38. Jacka FN, Cherbuin N, Anstey KJ, Butterworth P. Dietary patterns and depressive symptoms over time: examining the relationships with socioeconomic position, health behaviours and cardiovascular risk. PloS one. 2014;9(1):e87657.

39. Bakhtiyari M, Ehrampoush E, Enayati N, Joodi G, Sadr S, Delpisheh A, et al. Anxiety as a consequence of modern dietary pattern in adults in Tehran-Iran. Eating behaviors. 2013;14(2):107-12.

40. Weng T-T, Hao J-H, Qian Q-W, Cao H, Fu J-L, Sun Y, et al. Is there any relationship between dietary patterns and depression and anxiety in Chinese adolescents? Public health nutrition. 2012;15(4):673-82.

41. Li Y, Lv M-R, Wei Y-J, Sun L, Zhang J-X, Zhang H-G, et al. Dietary patterns and depression risk: A meta-analysis. Psychiatry research. 2017;253:373-82.

42. McMartin SE, Jacka FN, Colman I. The association between fruit and vegetable consumption and mental health disorders: evidence from five waves of a national survey of Canadians. Preventive medicine. 2013;56(3-4):225-30.

43. Mikolajczyk RT, El Ansari W, Maxwell AE. Food consumption frequency and perceived stress and depressive symptoms among students in three European countries. Nutrition Journal. 2009;8(1):31.

44. Meyer BJ, Kolanu N, Griffiths DA, Grounds B, Howe PR, Kreis IA. Food groups and fatty acids associated with self-reported depression: an analysis from the Australian National Nutrition and Health Surveys. Nutrition. 2013;29(7):1042-7.

45. Crichton GE, Murphy KJ, Bryan J. Dairy intake and cognitive health in middle-aged South Australians. Asia Pacific journal of clinical nutrition. 2010;19(2):161-71.

46. Miyake Y, Tanaka K, Okubo H, Sasaki S, Arakawa M. Intake of dairy products and calcium and prevalence of depressive symptoms during pregnancy in Japan: a cross-sectional study. BJOG: An International Journal of Obstetrics \& Gynaecology. 2015;122(3):336-43.

47. Rahman A, Baker PS, Allman R, Zamrini E. Dietary factors and cognitive impairment in community-dwelling elderly. The journal of nutrition, health \& aging. 2007;11(1):49.

48. Narmaki E, Siassi F, Koohdani F, Qorbani M, Shiraseb F, Ataie-Jafari A, et al. Dietary diversity as a proxy measure of blood antioxidant status in women. Nutrition. 2015;31(5):722-6.

49. Lopez-Garcia E, Schulze MB, Fung TT, Meigs JB, Rifai N, Manson JE, et al. Major dietary patterns are related to plasma concentrations of markers of inflammation and endothelial dysfunction. The American journal of clinical nutrition. 2004;80(4):1029-35.

50. Rumsfeld JS, Ho PM. Depression and cardiovascular disease: a call for recognition. Am Heart Assoc; 2005.

51. Zhang J-M, An J, inventorsCytokines, inflammation and pain2007.

52. Foote JA, Murphy SP, Wilkens LR, Basiotis PP, Carlson A. Dietary variety increases the probability of nutrient adequacy among adults. The Journal of nutrition. 2004;134(7):1779-85.

53. Mirmiran P, Azizi F. Determining the dietary diversity and its relation to dietary adequacy in teenagers of a district in Tehran. Research in Medicine. 2003;27(2):119-27.

54. Murakami K, Mizoue T, Sasaki S, Ohta M, Sato M, Matsushita Y, et al. Dietary intake of folate, other B vitamins, and $\omega$-3 polyunsaturated fatty acids in relation to depressive symptoms in Japanese adults. Nutrition. 2008;24(2):140-7.

55. Benton D, Donohoe RT. The effects of nutrients on mood. Public Health Nutrition. 1999;2(3a):403-9.

56. Payne JL. The role of estrogen in mood disorders in women. International Review of Psychiatry. 2003;15(3):280-90.

57. Marks GC, Hughes MC, van der Pols JC. Relative validity of food intake estimates using a food frequency questionnaire is associated with sex, age, and other personal characteristics. The Journal of nutrition. 2006;136(2):459-65.

\section{Tables}

Table 1. Factor loadings for the four extracted somatoform symptoms profiles. 


\begin{tabular}{|c|c|c|c|c|}
\hline \multirow[b]{2}{*}{ Somatoform symptoms } & \multicolumn{4}{|c|}{ Factor loadings $^{\mathrm{a}}$} \\
\hline & Psychological & Gastrointestinal & Neuro-skeletal & Pharyngeal-respiratory \\
\hline Sleep disorder & 0.420 & - & - & - \\
\hline Pounding heart & 0.577 & - & - & - \\
\hline Feeling low on energy & 0.565 & - & - & - \\
\hline Feeling like "butterflies" in the stomach & 0.869 & - & - & - \\
\hline Difficulty concentrating & 0.869 & - & - & - \\
\hline Disturbing thoughts & 0.664 & - & - & - \\
\hline Dry mouth & - & 0.248 & - & - \\
\hline Chest pain & - & 0.454 & - & - \\
\hline Feeling of fullness & - & 0.662 & - & - \\
\hline Nausea & - & 0.509 & - & - \\
\hline Gastroesophageal reflux & - & 0.544 & - & - \\
\hline Pain or discomfort in the abdomen & - & 0.678 & - & - \\
\hline Constipation & - & 0.512 & - & - \\
\hline Diarrhea & - & 0.374 & - & - \\
\hline Bloating or swelling of the abdomen & - & 0.644 & - & - \\
\hline Anal pain & - & 0.473 & - & - \\
\hline Headache & - & - & 0.547 & - \\
\hline Back pain & - & - & 0.661 & - \\
\hline Pain in joints & - & - & 0.612 & - \\
\hline Eyesore & - & - & 0.461 & - \\
\hline Severe fatigue & - & - & 0.646 & - \\
\hline Dizziness and confusion & - & - & 0.513 & - \\
\hline Chills and extreme cold & - & - & 0.435 & - \\
\hline Hot flashes & - & - & 0.350 & - \\
\hline Menstrual disorder & - & - & 0.389 & - \\
\hline Neck pain & - & - & - & 0.570 \\
\hline Globus sensation & - & - & - & 0.510 \\
\hline Having trouble swallowing & - & - & - & 0.596 \\
\hline Shortness of breath & - & - & - & 0.492 \\
\hline Hoarseness & - & - & - & 0.612 \\
\hline Wheezing (asthma) & - & - & - & 0.567 \\
\hline Variance explained (\%) & 12.70 & 11.73 & 11.55 & 8.85 \\
\hline Cumulative variance & 12.70 & 24.43 & 35.99 & 44.84 \\
\hline
\end{tabular}

${ }^{\mathrm{a}}$ Factor loadings $<0.2$ are not shown for simplicity.

Table 2. General characteristics of study participants across tertiles of dietary diversity score ${ }^{1}$.

\begin{tabular}{|c|c|c|c|c|}
\hline \multirow[b]{2}{*}{ Variables } & \multicolumn{3}{|c|}{ Dietary Diversity Score } & \multirow[b]{2}{*}{$\mathrm{P}_{\text {value }}{ }^{2}$} \\
\hline & 1 & 2 & 3 & \\
\hline Age (years) & $36.14 \pm 0.27$ & $36.39 \pm 0.26$ & $36.51 \pm 0.28$ & 0.615 \\
\hline BMI $\left(\mathrm{kg} / \mathrm{m}^{2}\right)$ & $25.12 \pm 0.14$ & $25.20 \pm 0.16$ & $24.84 \pm 0.13$ & 0.215 \\
\hline Male (\%) & 41.1 & 41.9 & 39.9 & 0.658 \\
\hline Married (\%) & 79.1 & 82.0 & 81.8 & 0.054 \\
\hline Educational level (\%) & & & & $<0.0001$ \\
\hline$\leq 12$ yrs & 12.7 & 11.1 & 8.2 & \\
\hline $12-16$ yrs & 81.1 & 80.9 & 81.6 & \\
\hline$>16$ yrs & 6.2 & 7.9 & 10.2 & \\
\hline Physically active (\%) & 29.9 & 30.5 & 41.3 & 0.585 \\
\hline Overweight or obese ${ }^{3}(\%)$ & 48.8 & 46.2 & 45.4 & $<0.0001$ \\
\hline Current smokers (\%) & 3.0 & 3.5 & 2.7 & 0.868 \\
\hline High score of Psychological symptoms (\%) & 53.4 & 50.1 & 45.5 & 0.004 \\
\hline High score of Gastrointestinal symptoms (\%) & 53.7 & 51.9 & 46.7 & 0.020 \\
\hline High score of Neuro-skeletal symptoms (\%) & 53.5 & 50.7 & 48.6 & 0.272 \\
\hline High score of Pharyngeal-respiratory symptoms (\%) & 49.1 & 47.2 & 40.9 & 0.001 \\
\hline
\end{tabular}

1 Values are Mean \pm SE unless otherwise indicated. BMI: body mass index.

2 Resulted from one-way ANOVA and $\chi^{2}$ test for continuous and categorical variables, respectively.

3 Overweight was defined as BMI greater than or equal to 25 and less than or equal to $29.99 \mathrm{~kg} / \mathrm{m}^{2}$ and obese was defined as BMI $\geq 30$ $\mathrm{kg} / \mathrm{m}^{2}$.

Table 3. Dietary intakes of participants across median cuts of somatoform symptoms profiles ${ }^{1}$. 


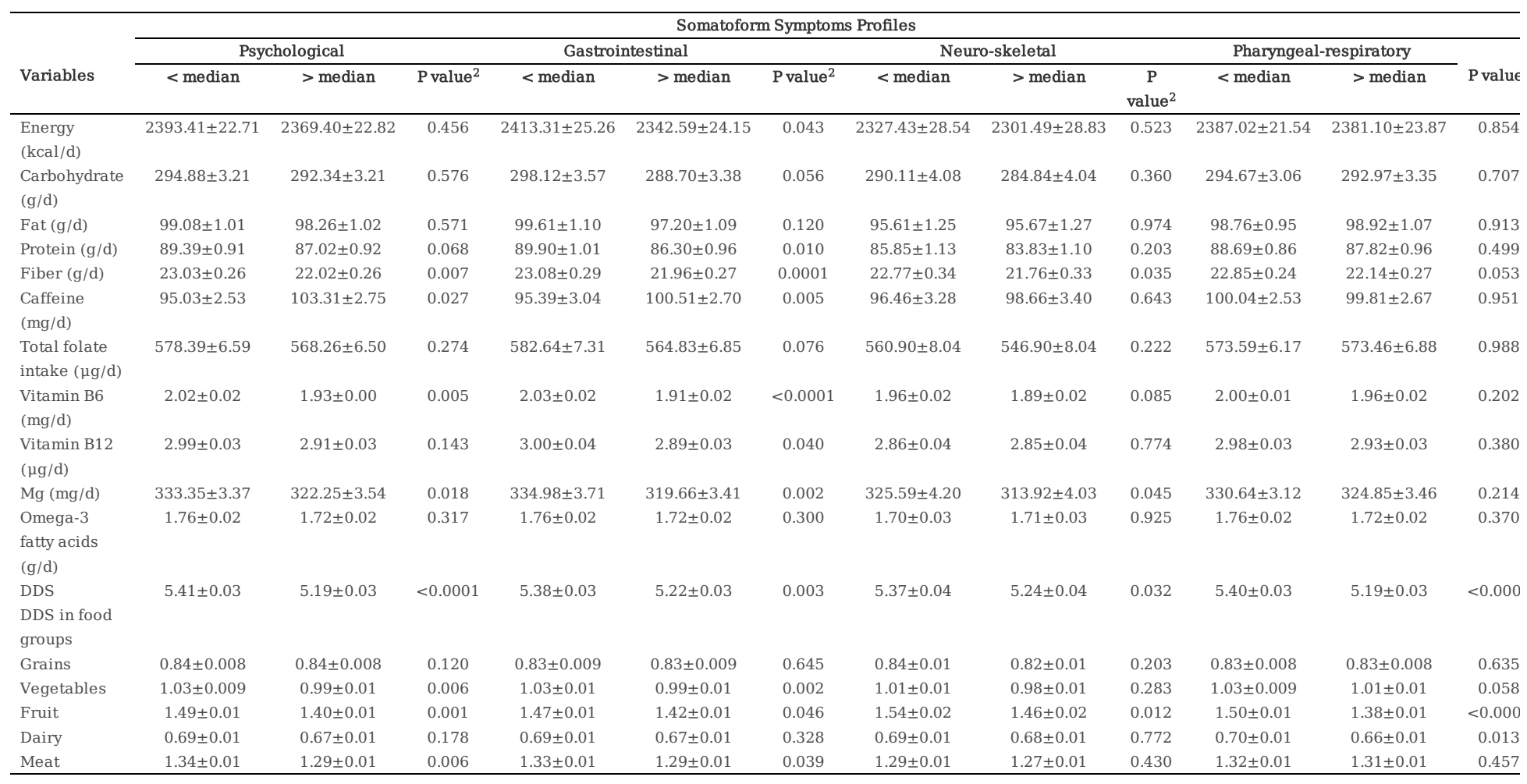

1 Values are Mean \pm SE. Nutrients were adjusted for age, sex and total energy intake (kcal). Energy intake was adjusted for age and sex.

2 From independent samples t-test and energy was considered as the absolute amount per day.

Table 4. Crude and multivariable-adjusted odds ratio and 95\% confidence interval for various psychosomatic symptoms profiles across tertiles of dietary diversity score.

\begin{tabular}{|c|c|c|c|c|}
\hline \multirow{2}{*}{ Psychosomatic symptoms profiles } & \multicolumn{3}{|c|}{ Tertiles of dietary diversity score } & \multirow{2}{*}{ P trend $^{1}$} \\
\hline & 1 & 2 & 3 & \\
\hline \multicolumn{5}{|l|}{ Psychological } \\
\hline Crude & $1.38(1.14,1.66)$ & $1.21(0.99-1.45)$ & 1 (Reference) & 0.004 \\
\hline Model 1 & $1.62(1.27,2.06)$ & $1.33(1.08,1.65)$ & 1 (Reference) & $<0.0001$ \\
\hline Model 2 & $1.56(1.18,2.05)$ & $1.32(1.04,1.67)$ & 1 (Reference) & 0.005 \\
\hline Model 3 & $1.44(1.06,1.95)$ & $1.35(1.04,1.75)$ & 1 (Reference) & 0.029 \\
\hline \multicolumn{5}{|l|}{ Gastrointestinal } \\
\hline Crude & $1.33(1.08-1.62)$ & $1.23(1.002-1.51)$ & 1 (Reference) & 0.02 \\
\hline Model 1 & $1.31(1.01,1.71)$ & $1.22(0.97,1.53)$ & 1 (Reference) & 0.090 \\
\hline Model 2 & $1.27(0.95,1.70)$ & $1.18(0.92,1.51)$ & 1 (Reference) & 0.221 \\
\hline Model 3 & $1.17(0.85,1.60)$ & $1.17(0.90,1.53)$ & 1 (Reference) & 0.444 \\
\hline \multicolumn{5}{|l|}{ Neuro-Skletal } \\
\hline Crude & $1.21(0.95,1.55)$ & $1.08(0.85,1.38)$ & 1 (Reference) & 0.273 \\
\hline Model 1 & $1.32(0.97,1.79)$ & $1.17(0.89,1.53)$ & 1 (Reference) & 0.198 \\
\hline Model 2 & $1.32(0.92,1.89)$ & $1.18(0.87,1.61)$ & 1 (Reference) & 0.288 \\
\hline Model 3 & $1.22(0.82,1.80)$ & $1.16(0.83,1.63)$ & 1 (Reference) & 0.548 \\
\hline \multicolumn{5}{|l|}{ Pharyngeal -Respiratory } \\
\hline Crude & $1.39(1.15,1.68)$ & $1.29(1.07,1.56)$ & 1 (Reference) & 0.001 \\
\hline Model 1 & $1.58(1.25,2.01)$ & $1.33(1.08,1.63)$ & 1 (Reference) & 0.001 \\
\hline Model 2 & $1.67(1.28,2.18)$ & $1.29(1.02,1.63)$ & 1 (Reference) & 0.001 \\
\hline Model 3 & $1.54(1.17,2.04)$ & $1.27(1.005,1.62)$ & 1 (Reference) & 0.009 \\
\hline
\end{tabular}

1 From Mantel-Haenszel extension chi-square test. 
Model 1: adjustment was made for age, sex and energy (kcal/d). Model 2: additional adjustment was made for marital status, education, smoking, physical activity and BMI. Model 3: anti-psychotic medicines, medical condition and stressful life events were additionally adjusted.

Table 5. Crude and multivariable-adjusted odds ratio and 95\% confidence interval for various psychosomatic symptoms profiles across tertiles of dietary diversity score stratified by sex. 


\begin{tabular}{|c|c|c|c|c|}
\hline \multirow{2}{*}{ Psychosomatic symptoms profiles } & \multicolumn{3}{|c|}{ Tertiles of dietary diversity score } & \multirow{2}{*}{ P trend ${ }^{1}$} \\
\hline & 1 & 2 & 3 & \\
\hline \multicolumn{5}{|l|}{ Male } \\
\hline \multicolumn{5}{|l|}{ Psychological } \\
\hline Crude & $1.35(0.99,1.83)$ & $1.31(0.97,1.78)$ & 1 (Reference) & 0.102 \\
\hline Model 1 & $1.33(0.89,1.97)$ & $1.28(0.90,1.81)$ & 1 (Reference) & 0.279 \\
\hline Model 2 & $1.37(0.88,2.12)$ & $1.30(0.89,1.91)$ & 1 (Reference) & 0.292 \\
\hline Model 3 & $1.13(0.69,1.85)$ & $1.35(0.88,2.07)$ & 1 (Reference) & 0.344 \\
\hline \multicolumn{5}{|l|}{ Gastrointestinal } \\
\hline Crude & $1.47(1.06,2.04)$ & $1.34(0.97,1.87)$ & 1 (Reference) & 0.055 \\
\hline Model 1 & $1.38(0.89,2.14)$ & $1.27(0.87,1.84)$ & 1 (Reference) & 0.297 \\
\hline Model 2 & $1.31(0.82,2.08)$ & $1.17(0.78,1.74)$ & 1 (Reference) & 0.502 \\
\hline Model 3 & $1.10(0.65,1.87)$ & $1.20(0.77,1.87)$ & 1 (Reference) & 0.708 \\
\hline \multicolumn{5}{|l|}{ Neuro-Skletal } \\
\hline Crude & $4.53(1.48,13.82)$ & $2.16(0.65,7.14)$ & 1 (Reference) & 0.024 \\
\hline Model 1 & $0.48(0.06,3.52)$ & $0.38(0.05,2.48)$ & 1 (Reference) & 0.598 \\
\hline Model 2 & $0.59(0.04,8.94)$ & $0.19(0.01,3.59)$ & 1 (Reference) & 0.528 \\
\hline Model 3 & $0.92(0.03,24.59)$ & $0.25(0.007,8.61)$ & 1 (Reference) & 0.674 \\
\hline \multicolumn{5}{|l|}{ Pharyngeal -Respiratory } \\
\hline Crude & $1.37(1.01,1.86)$ & $1.39(1.03,1.88)$ & 1 (Reference) & 0.050 \\
\hline Model 1 & $1.37(0.92,2.03)$ & $1.41(1.003,1.98)$ & 1 (Reference) & 0.122 \\
\hline Model 2 & $1.48(0.96,2.29)$ & $1.37(0.94,1.99)$ & 1 (Reference) & 0.151 \\
\hline Model 3 & $1.29(0.81,2.04)$ & $1.35(0.91,2.007)$ & 1 (Reference) & 0.305 \\
\hline \multicolumn{5}{|l|}{ Female } \\
\hline \multicolumn{5}{|l|}{ Psychological } \\
\hline Crude & $1.32(1.08,1.62)$ & $1.23(1.01,1.50)$ & 1 (Reference) & 0.020 \\
\hline Model 1 & $1.84(1.35,2.50)$ & $1.37(1.05,1.79)$ & 1 (Reference) & 0.001 \\
\hline Model 2 & $1.69(1.18,2.40)$ & $1.33(0.98,1.79)$ & 1 (Reference) & 0.013 \\
\hline Model 3 & $1.73(1.17,2.56)$ & $1.36(0.98,1.90)$ & 1 (Reference) & 0.020 \\
\hline \multicolumn{5}{|l|}{ Gastrointestinal } \\
\hline Crude & $1.26(0.96,1.64)$ & $1.17(0.89,1.52)$ & 1 (Reference) & 0.215 \\
\hline Model 1 & $1.29(0.93,1.78)$ & $1.19(0.90,1.58)$ & 1 (Reference) & 0.274 \\
\hline Model 2 & $1.27(0.89,1.80)$ & $1.24(0.91,1.68)$ & 1 (Reference) & 0.291 \\
\hline Model 3 & $1.20(0.81,1.77)$ & $1.16(0.83,1.62)$ & 1 (Reference) & 0.587 \\
\hline \multicolumn{5}{|l|}{ Neuro-Skletal } \\
\hline Crude & $1.14(0.88,1.46)$ & $1.05(0.81,1.35)$ & 1 (Reference) & 0.588 \\
\hline Model 1 & $1.34(0.98,1.82)$ & $1.19(0.91,1.57)$ & 1 (Reference) & 0.169 \\
\hline Model 2 & $1.32(0.91,1.89)$ & $1.21(0.89,1.66)$ & 1 (Reference) & 0.528 \\
\hline Model 3 & $1.20(0.80,1.78)$ & $1.18(0.84,1.65)$ & 1 (Reference) & 0.561 \\
\hline \multicolumn{5}{|l|}{ Pharyngeal -Respiratory } \\
\hline Crude & $1.42(1.11,1.81)$ & $1.24(0.97,1.58)$ & 1 (Reference) & 0.016 \\
\hline Model 1 & $1.72(1.27,2.31)$ & $1.28(0.98,1.66)$ & 1 (Reference) & 0.002 \\
\hline Model 2 & $1.81(1.29,2.55)$ & $1.23(0.92,1.66)$ & 1 (Reference) & 0.003 \\
\hline Model 3 & $1.75(1.22,2.49)$ & $1.21(0.89,1.64)$ & 1 (Reference) & 0.007 \\
\hline
\end{tabular}

1 From Mantel-Haenszel extension chi-square test.

Model 1: adjustment was made for age, sex and energy (kcal/d). Model 2: additional adjustment was made for marital status, education, smoking, physical activity and BMI. Model 3: anti-psychotic medicines, medical condition and stressful life events were additionally adjusted. 